of emerging therapies, such as ADCs, as well as identifying areas of continued educational need.

Disclosures Supported by an independent education grant from GenMab and Seattle Genetics.

Geoff Fisher, Amy Furedy, and Juliette Vandenbroucque have no relevant financial relationships.

Nicole Concin, has served as an advisor for AstraZeneca, Seattle Genetics and Mersana, and received travel expenses from Amgen, Genmab, and Roche.

\section{6 'ARE THERE LIMITS TO CURATIVE TREATMENTS?' - THE IMPACT OF CHEMO-RADIATION IN CERVICAL CANCER PATIENTS}

${ }^{1}$ Tiago Alpoim, ${ }^{2}$ Diana Ramada, ${ }^{2}$ Natividade Pais, ${ }^{3}$ Lurdes Salgado, ${ }^{3}$ Luísa Canvalho, ${ }^{1}$ Deolinda Pereira, 'Miguel Abreu. 'Instituto Português de Oncologia Do Porto; Medical Oncology; ${ }^{2}$ Instituto Português de Oncologia Do Porto; ${ }^{3}$ Instituto Português de Oncologia Do Porto; Radiation Oncology

\subsection{6/ijgc-2020-ESGO.23}

Introduction/Background Cervical cancer (CC) is the second most frequent gynaecologic tumour and curative treatment often includes concomitant chemo-radiation (RT/ChT). The aim of this study was to assess the early and late impact on quality of life (QOL) of patients treated with this treatment modality.

Methodology Prospective study of patients, treated in a tertiary cancer centre, with RT/ChT (weekly cisplatin) between 2014-2016 with a median follow-up of 54.7 months. QOL was assessed using validated versions of EORTC QLQ-C30 and QLQ-Cx24 questionnaires, looking for 7 principle domains: global health, role function, physical function, social function, financial issues, sexual function and symptoms. For this evaluation two distinct moments were defined: the first one at the first day of treatment and the second one after at least 3.7 years (min-max: 3.7-5.9 years). To avoid bias in long-term questionnaires' answers (moment two) there were excluded patients that had persistent disease after RT/ChT or recurred after complete response. Patients' answers were converted, by linear transformation, into 0-100 score intervals. Paired Sample T-test and Wilcoxon Signed Rank Test were used to compare results.

Results 50 patients were included, with average age at diagnosis of 52 years (24-74 years) and stage disease II (FIGO 2009 ) in 32 (64\%). First and second questionnaires were answered by 50 and 34 patients, respectively. There were no differences between the two moments concerning global health $(p=.41)$, role function $(p=.72)$, physical function $(p=.21)$, social function $(p=.86)$ and financial issues $(p=.21)$. An emotional improvement to second evaluation $(p=.03)$ and a decrease in cognitive function $(p=.007)$ were observed. Related to symptoms there were no differences, except for diarrhea that was worse $(p=.006)$ and lymphedema $(p=.005)$ that improved later in time. Although sexual dysfunction seemed similar $(p=.21)$, there were a progressive increase of sexual worry $(\mathrm{p}=.004)$.

Conclusion Careful assessment and handling of treatment toxicities is imperative to minimize long term sequelae of curative treatments. Particularly in these survivor's cohort, focus on cognitive and sexual problems and diarrhea seems extremely important.

Disclosures The authors declare no conflicts of interest.

\section{7 RADICAL HYSTERECTOMY IN EARLY CERVICAL CANCER: CHARACTERISTICS AND OUTCOMES OF A LARGE- CONTROLLED EUROPEAN POPULATION}

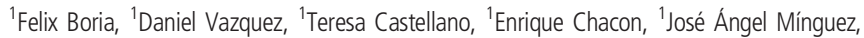
${ }^{2}$ Mauricio Cambeiro, ${ }^{3}$ Teresa Iscar, ${ }^{1}$ Antonio Gonzalez-Martin, ${ }^{1} J u a n$ Luis Alcazar, ${ }^{1}$ Luis M Chiva. 'Clínica Universidad de Navarra; ${ }^{2}$ Clinica Universidad de Navarra; Radiation Oncology; ${ }^{3}$ Clinica Universidad de Navarra; Pathology

\subsection{6/ijgc-2020-ESG0.24}

Introduction/Background Radical hysterectomy has been for years the primary treatment for early cervical cancer.

Recently, a number of retrospective studies and a prospective randomized trial (LACC trial) revealed higher rates of recurrence and death in patients that underwent minimally invasive surgery.

In Europe, we did not counted with large-contrasted data. We designed the SUCCOR study, a multicenter, observational cohort study with the primary goal of validating the results of the LACC trial in Europe.

In this study we try to compile all the information obtained in the Succor study to offer a comprehensive picture of the characteristics and outcomes of the surgical treatment of early cervical cancer in this large European population.

Methodology Patients were eligible if they underwent a radical hysterectomy in a European Institution for stage IB1 cervical cancer (FIGO 2009), from January 1st, 2013 to December 31st, 2014.

Results From May 15th to November 15th, 2019, we received data from a total of 1272 patients. 116 patients were excluded. The final cohort was composed of 1156 patients.

The median age of the studied population was 47.12 yo (10.8). 36.6\% of patients had undergone a cone biopsy before the radical hysterectomy. The mean preoperative maximum diameter measured by magnetic resonance imaging was 19.58 $\mathrm{mm}$ (13.3).

$633(54 \%)$ radical hysterectomies were done by laparotomy and all the other $523(46 \%)$ by minimally invasive surgery.

Nerve sparing technique was carry out in $61.8 \%$ of cases. Only in 224 cases (19.4\%) the sentinel lymph node biopsy was performed with a rate of bilateral identification of $79.7 \%$.

The average length of stay in the hospital was 6.72 days (4.2).

The average number pelvic nodes retrieved was 25.51, showing $12.4 \%$ of patients, nodal metastasis.

510 patients (44.1) received any type of adjuvant therapy. Standard radiation plus braquitherapy were the most frequent used modality of adjuvant treatment (54.1 and 43.3\% respectively) while concomitant chemoradiation was use in $34.1 \%$ of the cases.

After a median follow up of 58 months, the 5 -y disease free survival rate was $88.3 \%$ and the overall survival rate at 5 -y was 94.9\%.

Conclusion In this study we have collected the most extensive amount of information ever obtained on radical hysterectomy for cervical cancer in our continent. Even though it is a retrospective registry, the meticulous design of the inclusion and exclusion criteria offers a high level of accuracy when evaluating the surgical outcome of patients with cervical cancer after radical hysterectomy.

Disclosures All authors contributed to writing the manuscript and read and approved the final manuscript.

The authors declare no conflict of interest. 